Año 13.

Núm. 34 especial. Tema COVID 19.

ISSN: 2007-8870

https://revistainvestigacionacademicasinfrontera.unison.mx/index.php/RDIASF

Recibido el 14 de abril de 2020. Dictaminado mediante arbitraje favorablemente 2 de septiembre de 2020.

\title{
El impacto de la crisis sanitaria generada por COVID-19 en la finanzas de las Pequeñas y medianas empresas (Pymes) de Hermosillo, Sonora.
}

\author{
Dr. Martín Guillermo Durán Acosta \\ martin.duran@unison.mx \\ https://orcid.org/0000-0002-8716-7713 \\ Universidad de Sonora, Unidad Centro
}

\begin{abstract}
Resumen
Esta investigación, por diseño, es de tipo descriptiva y exploratoria y su objetivo es determinar, a partir de la apreciación que tienen los gerentes o responsables de la gestión financiera de las Pymes, como impacta a sus finanzas la crisis de salud ocasionada por el COVID-19 para el desarrollo e inverción de sus negocios. Los resultados obtenidos en la investigación muestran que la crisis de salud provocada por el COVID-19 ha sido un desafío para las Pymes porque ha generado una fuerte crisis, pero se han mostrado cautelosas en las medidas para enfrentarla, y las estrategias de gestión financiera orientadas a evitar el endeudamiento. En conclusión, la gerencia de las Pymes es consciente de que para afrontar la nueva modalidad es necesario realizar una gestión administrativa y financiera basadas en estrategias previsibles, teniendo en cuenta los cambios encaminados al desarrollo e inversión de sus negocios.
\end{abstract}

Palabras Clave: Gestión Financiera, COVID-19, Crisis Sanitaria, Desafío, Pymes, Impacto. 


\title{
The impact of the health crisis generated by COVID-19 on the finances of Small and medium-sized enterprises (SMEs) in Hermosillo, Sonora
}

\begin{abstract}
This research, by design, is descriptive and exploratory and its objective is to determine, based on the appreciation that managers or those responsible for the financial management of SMEs have, how it impacts the health crisis caused by the COVID-19 for the development and investment of their businesses. The results obtained in the investigation show that the health crisis caused by COVID-19 has been a challenge for SMEs because it has generated a strong crisis, but they have been cautious in the measures to face it, and financial management strategies aimed at avoiding the indebtedness. In conclusion, the SME management is aware that to face the new modality it is necessary to carry out administrative and financial management based on foreseeable strategies, taking into account changes aimed at the development and investment of their businesses.
\end{abstract}

Key words: Financial Management, COVID-19, Health Crisis, Challenge, SMEs, Impact..

\section{Introducción}

Las micro, pequeñas y medianas empresas (mipymes) le dan vida a las economías de América Latina y el Caribe (ALC), representan 99,5\% de todas las empresas de la región y $60 \%$ de la población ocupada y son responsables por el $25 \%$ del producto interno bruto (PIB) regional, según datos de la Organización para la Cooperación y el Desarrollo Económico (OECD, 2019), pero a pesar de su importancia económica y social, a estas empresas les resulta difícil acceder a financiamiento. De forma agregada, el mismo estudio menciona que la brecha entre la demanda y la oferta de crédito para mipymes en América Latina y el Caribe llega a ser de US\$1,8 mil millones, equivalentes al 41\% del PIB regional, de acuerdo al SME Finance Forum (2019) y además, indican que aproximadamente el 47\% de las pequeñas empresas (5 a 19 trabajadores) en el sector formal, no tienen un préstamo bancario o una línea de crédito y si la empresa es propiedad de una mujer, este número llega a más del 50\% (Herrera, 2020). 
Año 13.

Revista de Investigación

Núm. 34 especial. Tema COVID 19.

Académica sin Frontera

https://revistainvestigacionacademicasinfrontera.unison.mx/index.php/RDIASF

Recibido el 14 de abril de 2020. Dictaminado mediante arbitraje favorablemente 2 de septiembre de 2020.

A partir de la pandemia generada por el COVID-19, con sus estrepitosas consecuencias económicas, ha generado una problemática financiera que ha llevado a muchos negocios a cerrar o, en el mejor de los casos, a replantearse su estructura. Se puede observar que los gobiernos de algunos países de Latinoamérica, han empezado a anunciar algunas medidas de auxilio para las pymes en medio de cuarentena y de una paralización de la actividad comercial en algunos casos casi nula resultado esta situación un desafio para las finanzas de los negocios.

Actualmente las economías latinoamericanas experimentan los inicios de una recesión económica inminente, la cual de no ser tratada de manera correcta podría llegar a ser una de las más desastrosas a lo largo de la historia debido al impacto que tiene en distintos países (Gráfico 1). El Fondo Monetario Internacional (16 de abril de 2020) advirtió que "los países de Latinoamérica se enfrentan ante lo que posiblemente será otra década perdida entre el período comprendido de 2015 a 2025, debido a los negativos factores económicos y financieros que se han presentado en los últimos años y que ahora se potenciaron con la pandemia del coronavirus."

Gráfico 1. América Latina: Tasa de variación del PIB, 1901 a 2020 (porcentajes)

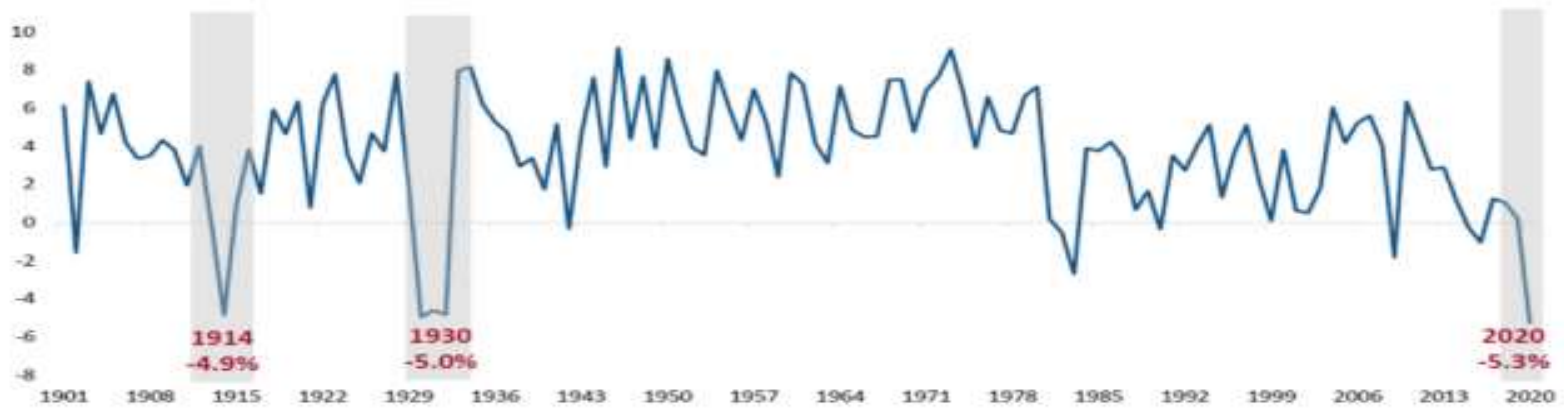

Fuente: CEPAL, sobre la base de datos de Maddison (2018).

La literatura señala como principales restricciones para el acceso a financiamiento por parte de las pymes: fallas del mercado, las características de la conformación de la oferta de crédito y las 
metodologías utilizadas por las entidades financieras para la evaluación y estrategias de proyectos (Urquía, Pérez y Muñoz, 2011). Por su parte Goldstein (2011), plantea que la problemática puede darse si la falta de oferta de créditos a las pymes es el resultado de una demanda reducida por parte de las empresas o si, en cambio, se debe a restricciones de la oferta (Goldstein, 2011). En el mismo orden de ideas, Ferraro y Goldstein (2011), señalan que la cantidad de recursos canalizados, también se ve influida por el método de selección de beneficiarios que se aplique, este depende, por un lado, de la información que se encuentra disponible, y, por el otro, de las características de las entidades que operan en el mercado y las estrategias de gestión financieras utilizadas en la competitividad empresarial.

Es indudable que para comprender los sistemas financieros recientes, y primero se debe analizar los datos mas recientes disponibles sobre la situación antes de la pandemia del Covid-19, Según cifras del Fondo Monetario Internacional (FMI). El panorama es desolador: la proyección es que la economía mexicana, se contraerá un 5,2\%, y es importante tomar en consideración que las pymes son empresas que dependen de las ventas diarias y tienen escasas o nulas reservas de liquidez, también pueden verse sometidas a un grave estrés financiero y es posible que tengan que despedir trabajadores, tal y como lo describe un informe publicado el 9 de abril del 2020 por el Banco Interamericano de Desarrollo (BID).

a pandemia provocada por el COVID-19, ha generado una problemática en materia económica y ha deteriorado de forma importante las perspectivas que tenían las pymes de lograr un crecimiento. El Fondo Monetario Internacional (FMI, 2020) y la Comisión Económica para América Latina y el Caribe (CEPAL, 2020) estiman que el crecimiento de México durante 2020 será de alrededor de $-6.6 \%$; como consecuencia de las cuarentenas implementadas, debido al menor envío de remesas, los menores precios de las materias primas, los menores ingresos por turismo, la mayor aversión al riesgo por parte de los inversionistas internacionales, las salidas de capitales y la mayor volatilidad en los mercados financieros.

Las expectativas futuras son toda una incógnita, ya que la crisis del COVID-19 tiene a los consumidores y las empresas en una incertidumbre continua, además, indica que algunos indicadores, como el Índice de Gerentes de Compras (Purchasing Manager Index - PMI -), han bajado drásticamente y que a partir del PMI se puede determinar si una economía goza de una buena salud, y tomar medidas de forma previa si los datos indican una posible recesión, por eso este indicador es especialmente importante para poder aventurarse a una 
Año 13.

Revista de Investigación

Núm. 34 especial. Tema COVID 19.

https://revistainvestigacionacademicasinfrontera.unison.mx/index.php/RDIASF

Recibido el 14 de abril de 2020. Dictaminado mediante arbitraje favorablemente 2 de septiembre de 2020.

crisis económica y reducir su impacto impulsando medidas, ya que los datos del PMI son utilizados por los profesionales financieros para comprender cuál es el rumbo y las expectativas de cada economía (Owen, 2020).

Pese al tamaño del impacto de la pandemia en la economía, México es uno de los países de América Latina y el mundo que menos recursos ha destinado al apoyo del sector privado y, de acuerdo con un análisis de la Comisión Económica para América Latina y el Caribe (CEPAL, 2020), el gobierno mexicano ha destinado apoyos y créditos para las empresas y negocios equivalentes a 3.8\% del PIB, comparado con Chile, que ha destinado 11.4\%, Colombia 8\%, Perú $7.6 \%$, Uruguay $5.3 \%$ y Costa Rica $4.3 \%$, teniendo esta estrategia del gobierno mexicano, claras consecuencias en la situación financiera de las pymes.

La pandemia del COVID-19 generó una crisis económica y sanitaria sin precedentes y a una escala, literalmente, global donde América Latina y el Caribe son una de las regiones más afectadas, y sus estructuras productivas y sociales la exponen a la mayor crisis económica de su historia, donde la situación del sector empresarial depende del rol que desempeñen los organismos internacionales de crédito en el marco de sus mandatos y misiones (Gasparini, Molinari y Patrucchi, 2020).

El éxito de la empresa depende de la forma en que enfrentes los desafios, porque uno de los errores más comunes es no darse la oportunidad de entender el contexto y naturaleza de la crisis y por ende sus alcances, esto implica la imposibilidad de preparse para algo que no se comprende. Hay que analizar los riesgos en las operaciones, finanzas y el modelo de negocios de la empresa ya que en una crisis como es la pandemia se deben considerar las oportunidades que se presenten y ya sea continuar trabajando como se venía operando, lo cual no es la realidad, o innovar nuevos procedimientos a través de un riguroso análisis y nueva planeación adecuada a los tiempos de incertidumbre (Pantaleón, 2020) 
Basado en lo anterior, se deduce la importancia del presente trabajo de investigación, debido a que el resultado del proyecto ayudará a cumplir con el objetivo de determinar, a partir de la apreciación que tienen los gerentes o responsables de la gestión financiera de las Pymes, como impacta a sus finanzas la crisis de salud ocasionada por el COVID-19 para el desarrollo e inverción de sus negocios.

\section{Materiales y Método}

En este documento se presentan los resultados de una investigación de campo bajo un enfoque metodológico de tipo descriptiva y exploratoria y su objetivo es determinar, a partir de la apreciación que tienen los gerentes o responsables de la gestión financiera de las Pymes del sector comercio de Hermosillo, Sonora, como impacta a sus finanzas la crisis de salud ocasionada por el COVID-19 para el desarrollo e inverción de sus negocios. Para este estudio se aplicó un cuestionario estandarizado (validado a juicio de expertos y con un coeficiente de confiabilidad Alpha de Cronbach de 0,92 Muy Alta) por (González-Díaz \& Perez, 2015) con 10 items a 50 gerentes de Pymes ubicadas en la ciudad de Hermosillo, Sonora, pertenecientes al sector comercio y una antigüedad entre tres y siete años de actividad en el mercado mediante un Google form (González-Díaz et al., 2016).

\section{Resultados}

Se presentan a continuación los resultados más relevantes de los avances de la investigación basados en el objetivo central del estudio: 
Año 13.

Núm. 34 especial. Tema COVID 19.

ISSN: 2007-8870

https://revistainvestigacionacademicasinfrontera.unison.mx/index.php/RDIASF

Recibido el 14 de abril de 2020. Dictaminado mediante arbitraje favorablemente 2 de septiembre de 2020.

Imagen 1. Impacto de la crisis sanitaria provocada por el COVID-19.

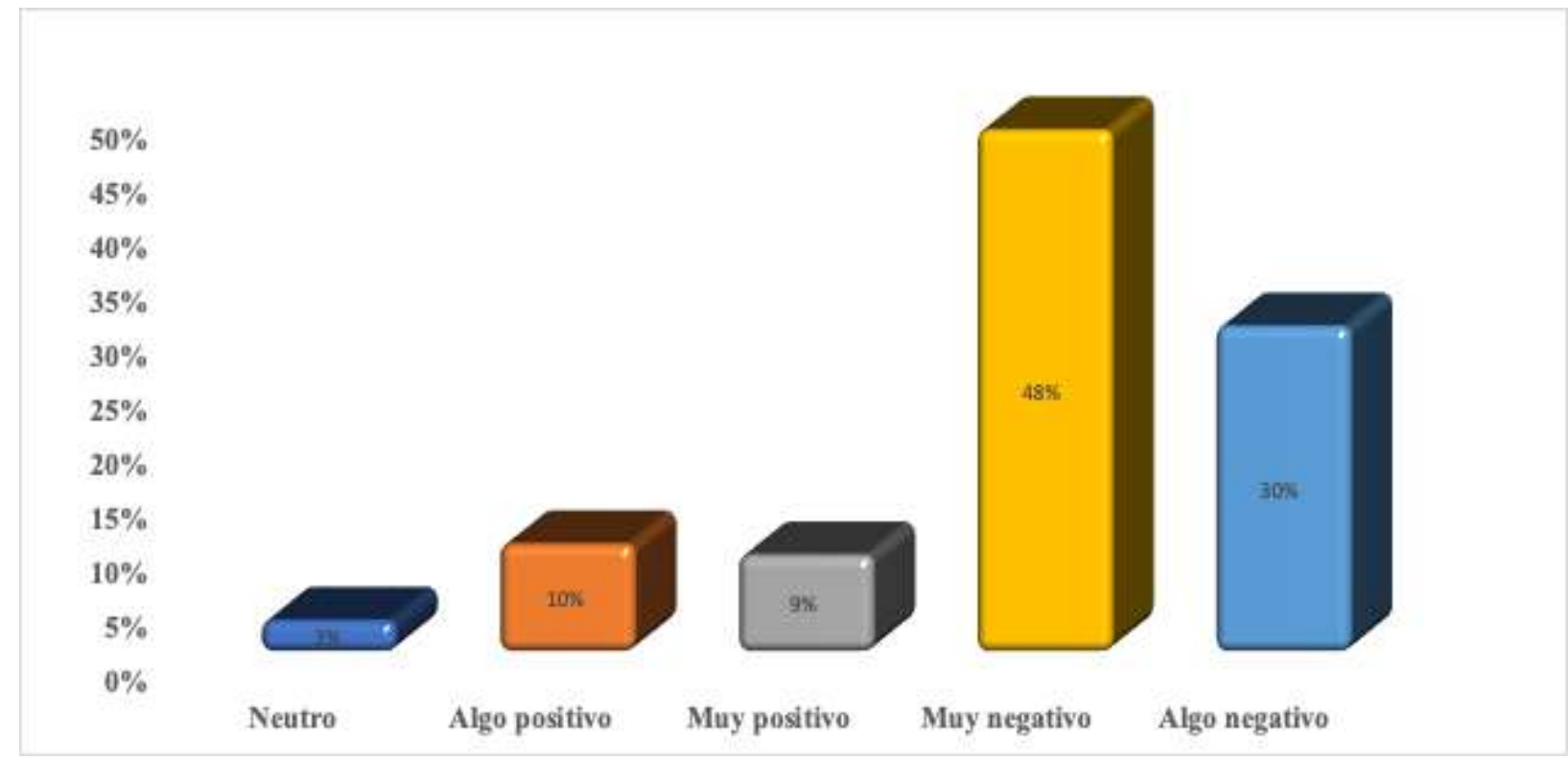

Fuente: Elaboración propia en base a resultados de la investigación.

La imagen 1, muestra que para el $46 \%$ de las Pymes del sector comercio minoristas de la ciudad de Hermosillo, Sonora el impacto del COVID-19 ha sido muy negativo, para el 32\% ha sido algo positivo, para el $10 \%$ ha sido algo positivo, para el $9 \%$ muy positivo y para un $3 \%$ es neutro. Los resultados hacen ver que la situación de las Pymes del sector comercio durante la contingencia generada por el COVID-19 se ha visto afectada. 
Imagen 2. Actividad económica durante el período del 20 de marzo al 20 de junio de 2020

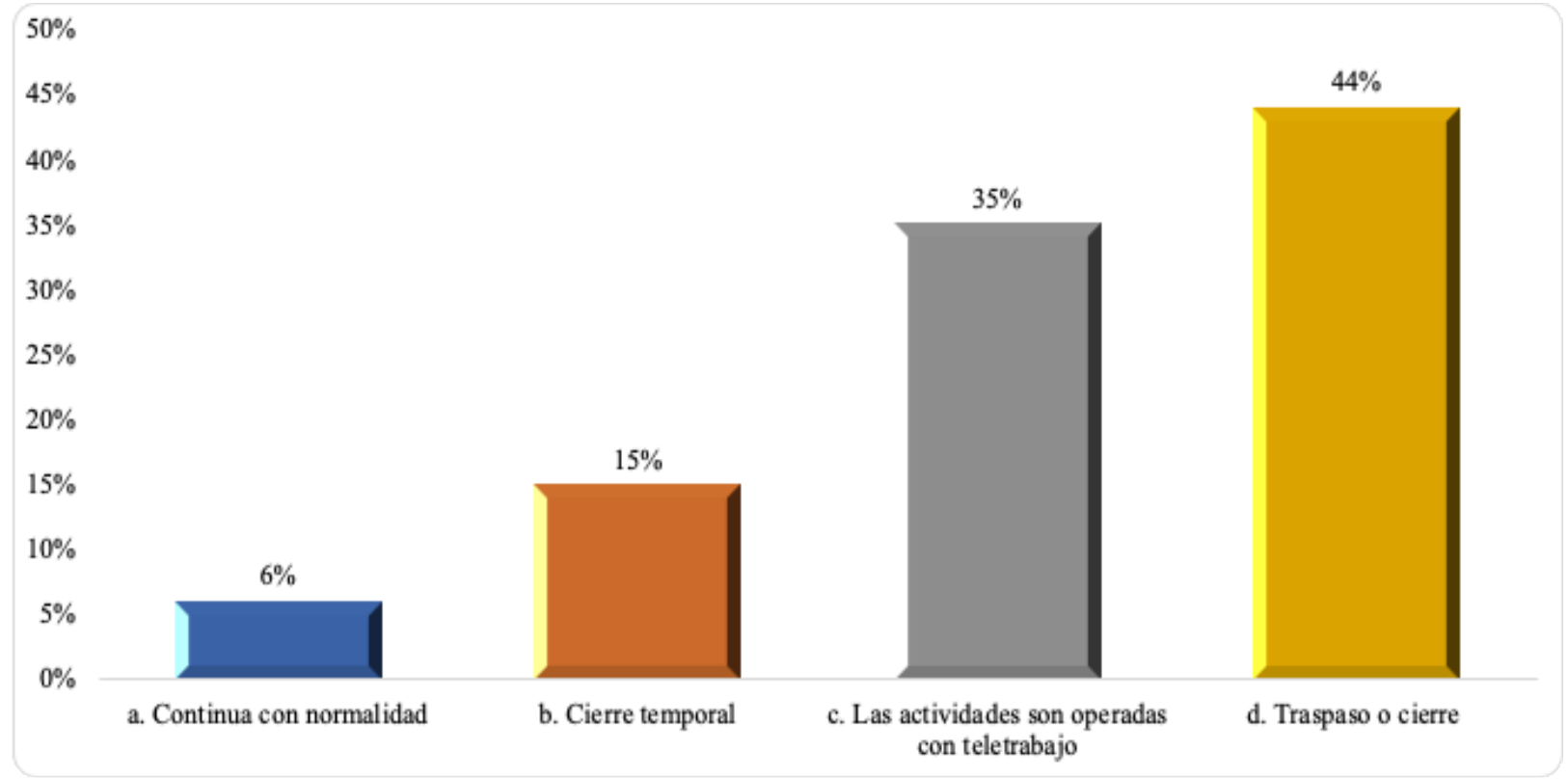

Fuente: Elaboración propia en base a resultados de la investigación.

La imagen 2, muestra que para el $46 \%$ de las Pymes del sector comercio minoristas de la ciudad de Hermosillo, Sonora el impacto del COVID-19 ha sido muy negativo, para el 32\% ha sido algo positivo, para el $10 \%$ ha sido algo positivo, para el 9\% muy positivo y para un 3\% es neutro. Los resultados hacen ver que la actividad económica durante el período del 20 de marzo al 20 de junio de 2020 de las Pymes del sector comercio en la ciudad de Hermosillo, Sonora durante la contingencia generada por el COVID-19 se ha visto afectada, lo anterior permite suponer que para poder enfrentarla se replantearon las estrategias de gestión financiera. 
Año 13.

Núm. 34 especial. Tema COVID 19.

ISSN: 2007-8870

https://revistainvestigacionacademicasinfrontera.unison.mx/index.php/RDIASF

Recibido el 14 de abril de 2020. Dictaminado mediante arbitraje favorablemente 2 de septiembre de 2020.

Imagen 3. Principales problemáticas

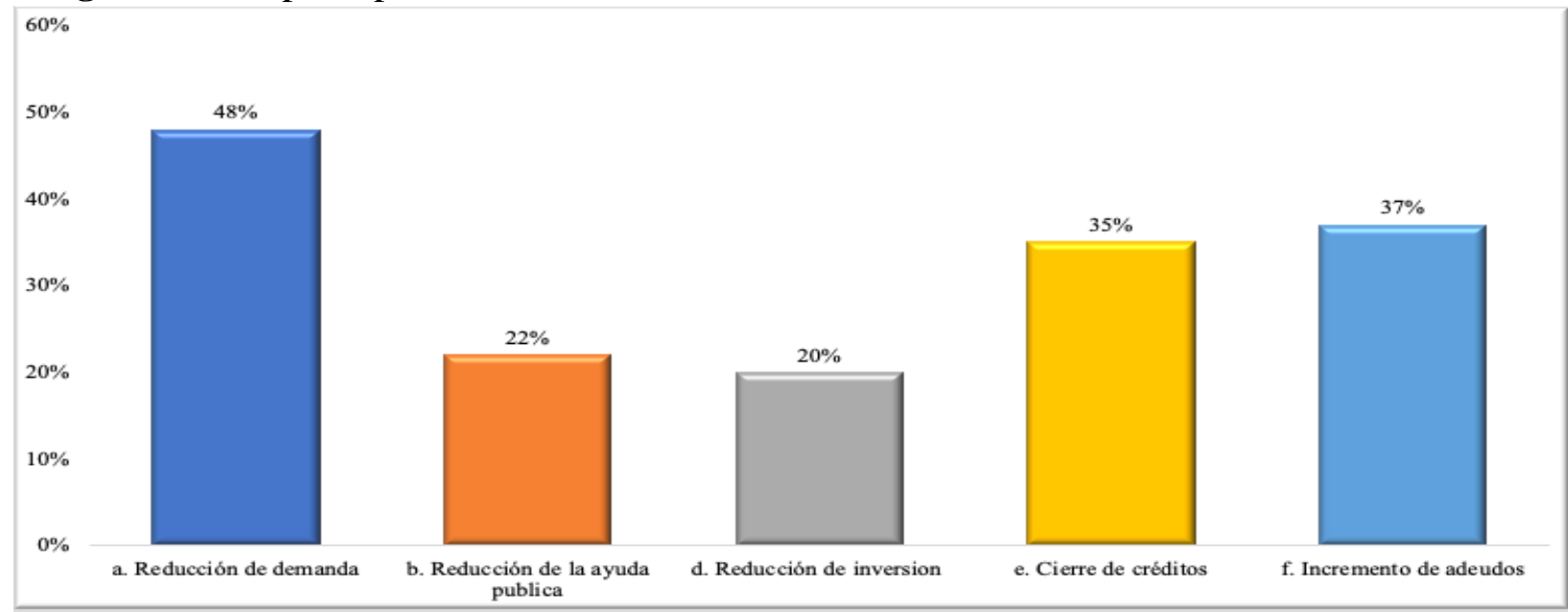

Fuente: Elaboración propia en base a resultados de la investigación.

La imagen 3, muestra que para el $48 \%$ de las Pymes del sector comercio de la ciudad de Hermosillo, Sonora el impacto del COVID-19 su principal problemática generada fue la reducción de la demanda, para el 37\% ha sido el incremento de sus adeudos, para el 35\% ha sido el cierre de créditos, para el 22\% la reducción de la ayuda pública y para un $20 \%$ es la reducción de inversión. Los resultados muestran que la principal problemática de las Pymes del sector comercio durante la contingencia generada por el COVID-19 se ha visto afectada especialmente por la reducción de la demanda y los incrementos de sus deudas y los cierres de las aperturas de apoyo financiero para poder enfrentarlas. 
Imagen 4. Estrategias de gestión financiera adoptadas a partir de la pandemia de COVID-19

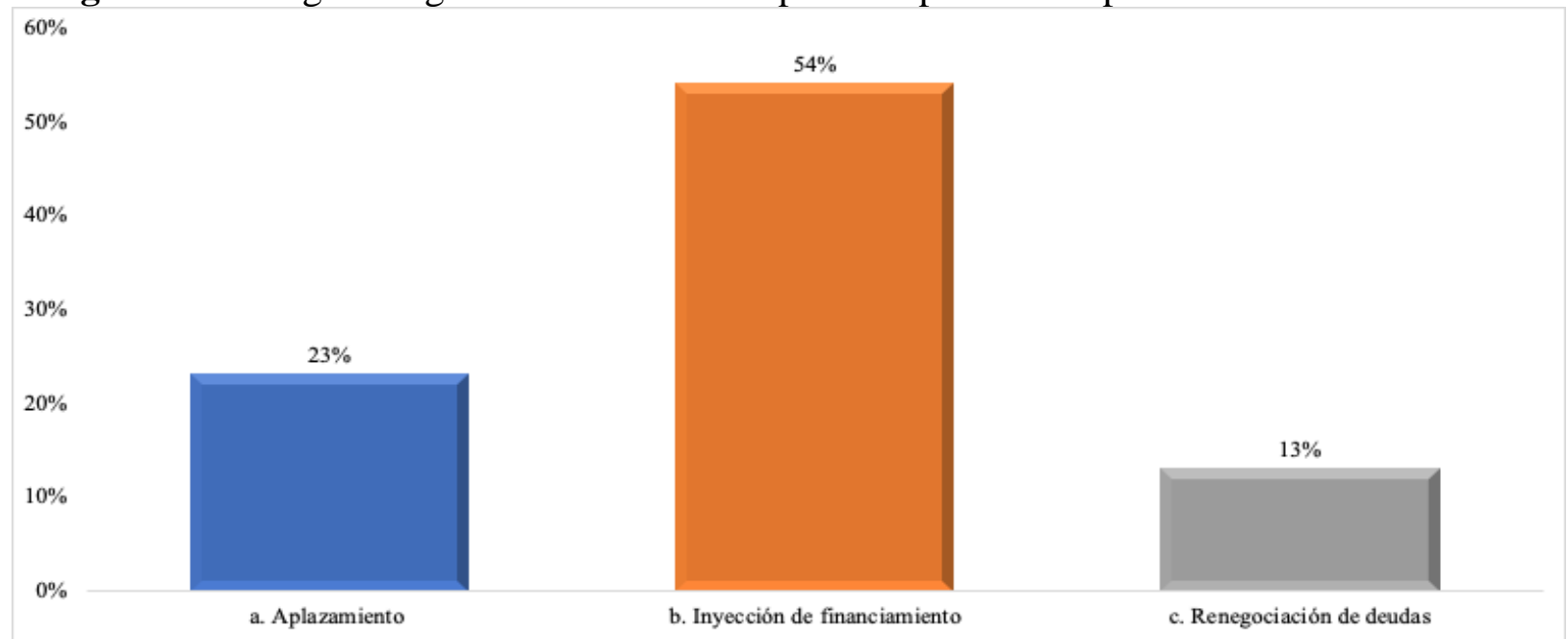

Fuente: Elaboración propia en base a resultados de la investigación.

La imagen 4, muestra que para el 54\% de las Pymes del sector comercio de la ciudad de Hermosillo, Sonora adoptó inyección de financiamiento como estrategias de gestión financieras, el $23 \%$ su estrategia de gestión financiera adoptada es el aplazamiento y el 13\% la renegociación de la deuda. Los resultados muestran que el empresario busca conservar su negocio, lo anterior permite suponer que buscó nuevas y variadas formas de financiamiento para conservar su negocio.

Imagen 5. Tipo de financiamiento utilizado para enfrentar el desafío de la crisis por COVID-19

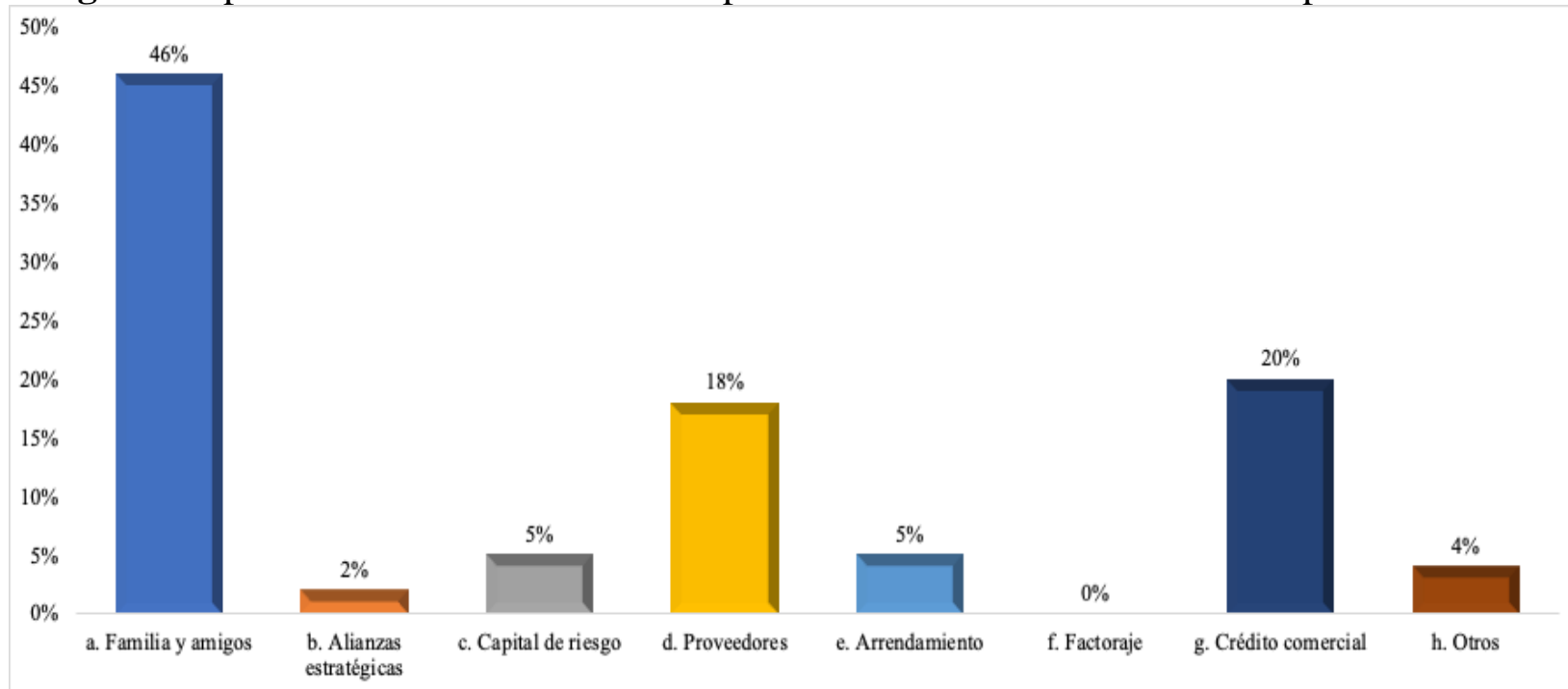

Fuente: Elaboración propia en base a resultados de la investigación. 
Año 13.

Núm. 34 especial. Tema COVID 19.

ISSN: 2007-8870

https://revistainvestigacionacademicasinfrontera.unison.mx/index.php/RDIASF

Recibido el 14 de abril de 2020. Dictaminado mediante arbitraje favorablemente 2 de septiembre de 2020.

La imagen 5, muestra que para el $46 \%$ de las Pymes del sector comercio de la ciudad de Hermosillo, Sonora el tipo de financiamiento utilizado para enfrentar el desafío de la crisis ha sido la familia y amigos, para el $20 \%$ ha sido el crédito comercial, para el $18 \%$ los proveedores, para el 5\% el capital de riesgo y el arrendamiento, para el $4 \%$ otros y para un $2 \%$ las alianzas. Los resultados muestran que la principal medida de gestión financiera para enfrentar el desafío de la crisis por parte de las Pymes fue la propia familia y amigos, lo anterior hace suponer que el empresario fue cauto en medidas de endeudamiento.

Imagen 6. Perfil del riesgo para enfrentar la crisis desde la perspectiva del gerente Pyme, considerando 1 el menos y 9 el mas relevante

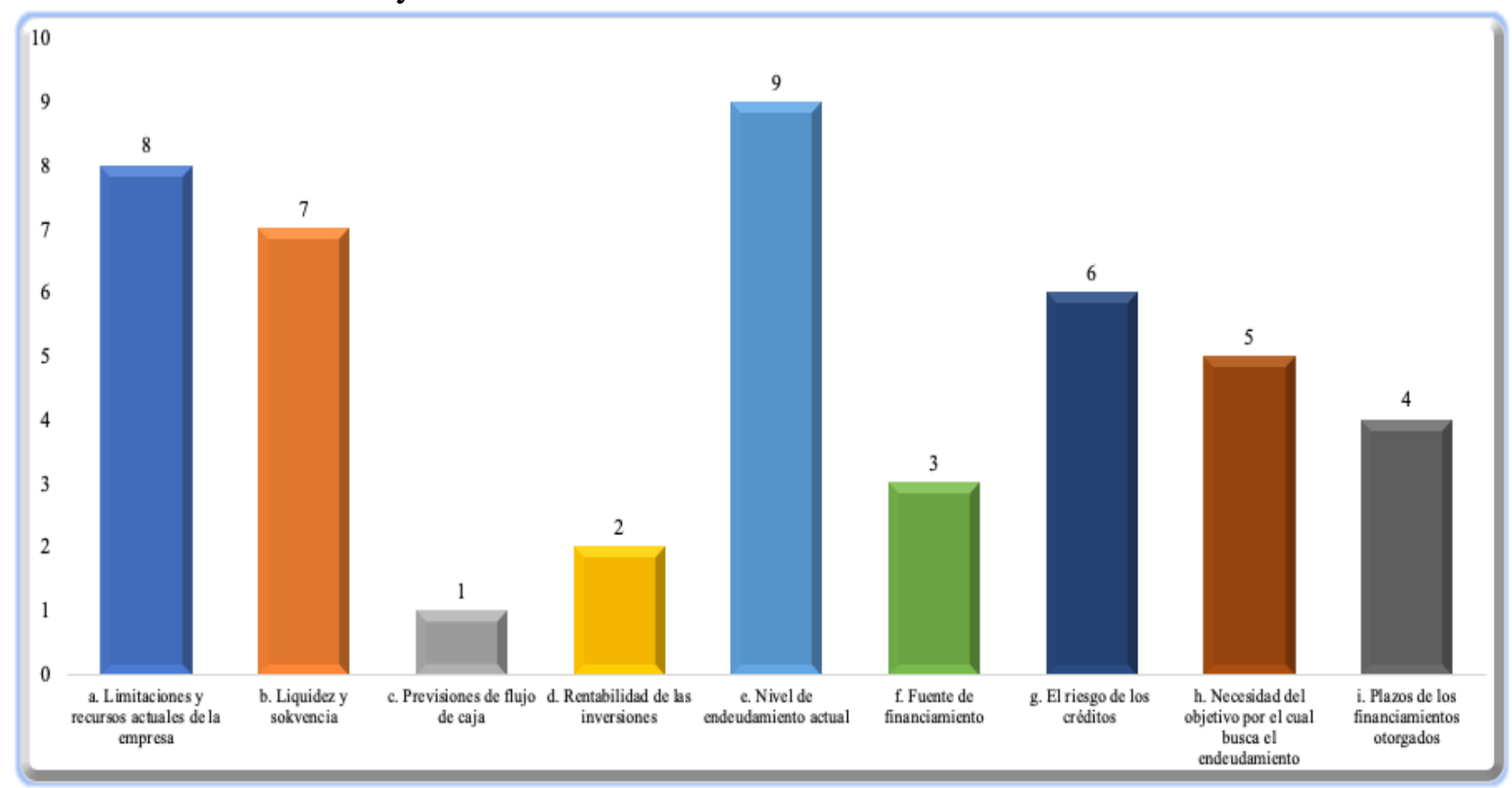

Fuente: Elaboración propia en base a resultados de la investigación.

La imagen 6, muestra que para el gerente de Pyme, al momento de hacer un perfil de riesgo para enfrentar la crisis su prioridad mas importante es conocer el nivel de endeudamiento actual y, lo menos importante son las previsiones de flujo de caja. Considerando la importancia 
de este perfil se observa en segundo lugar del perfil están las limitaciones y recursos actuales de la empresa, en tercero la liquidez y solvencia de la empresa, en cuarto lugar está el riesgo de los créditos y en quinto lugar la necesidad del objetivo para el cual busca el endeudamiento. Los resultados muestran que el empresario está mostrando cautela en el caso del endeudamiento, lo anterior hace suponer que el empresario esta consciente de que la rentabilidad de su negocio en momento de crisis no puede sustentar una inversión de momento porque esta esta orientada a aumentar el valor de la empresa y el panorama es aun muy incierto.

\section{Imagen 7. Expectativas a corto plazo}

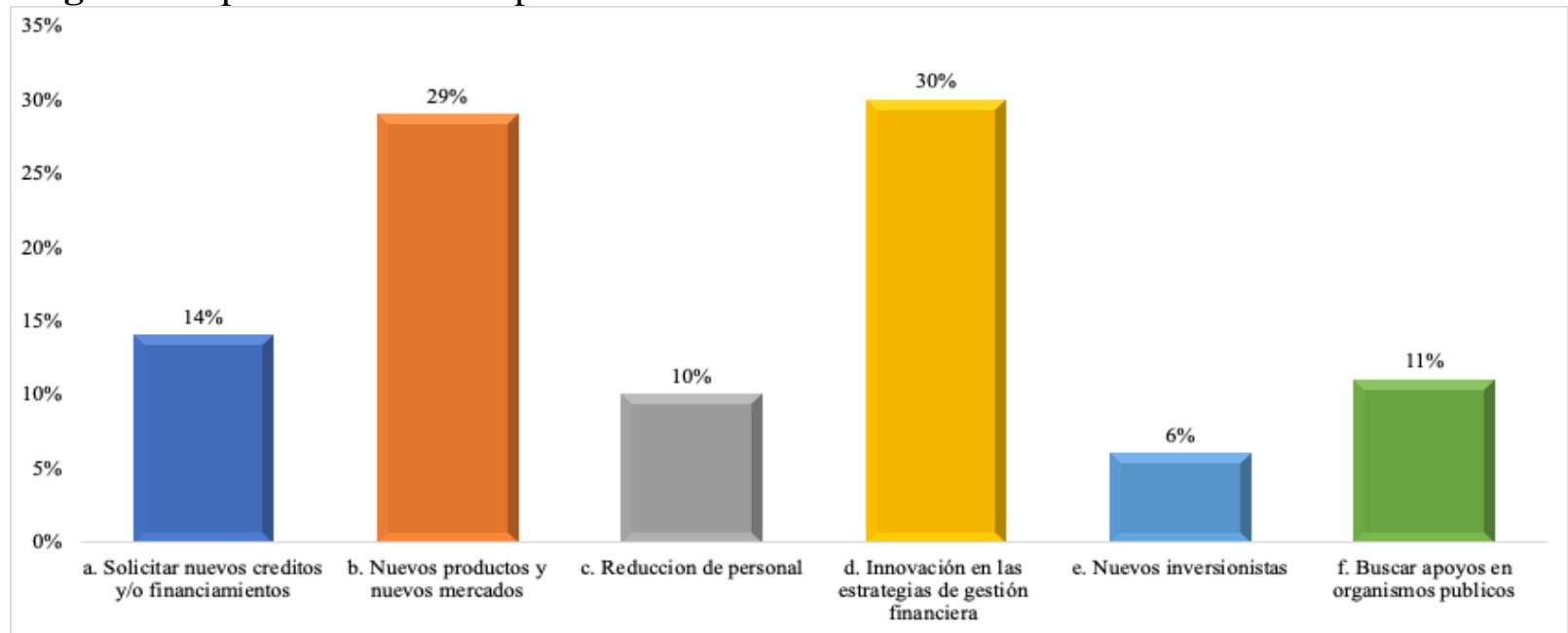

Fuente: Elaboración propia en base a resultados de la investigación.

La imagen 7, muestra para el $30 \%$ de la muestra analizada, las principales expectativas a corto plazo son innovación en las estrategias de gestión financiera, el 29\% nuevos productos y nuevos mercados, el $14 \%$ sus expectativas son solicitar nuevos créditos y/o financiamientos, el $11 \%$ buscar apoyos en organismos públicos, el 10\% reducción de personal y el $6 \%$ nuevos inversionistas. Los resultados obtenidos muestran que el empresario Pyme está consciente de la necesidad de crear innovación en sus estrategias de gestión financiera y de generar nuevos productos y nuevos mercados, lo anterior hace suponer que seguirá buscando la posibilidad tal vez de reinventarse en sus estrategias de gestión administrativas para lo cual es importante la información financiera que se pueda proporcionar de parte del área de finanzas. 
Año 13.

Núm. 34 especial. Tema COVID 19.

ISSN: 2007-8870

https://revistainvestigacionacademicasinfrontera.unison.mx/index.php/RDIASF

Recibido el 14 de abril de 2020. Dictaminado mediante arbitraje favorablemente 2 de septiembre de 2020.

Imagen 8. Si COVID-19 terminara hoy, ¿cuánto tiempo le tomaría a tu empresa "volver a la normalidad"?

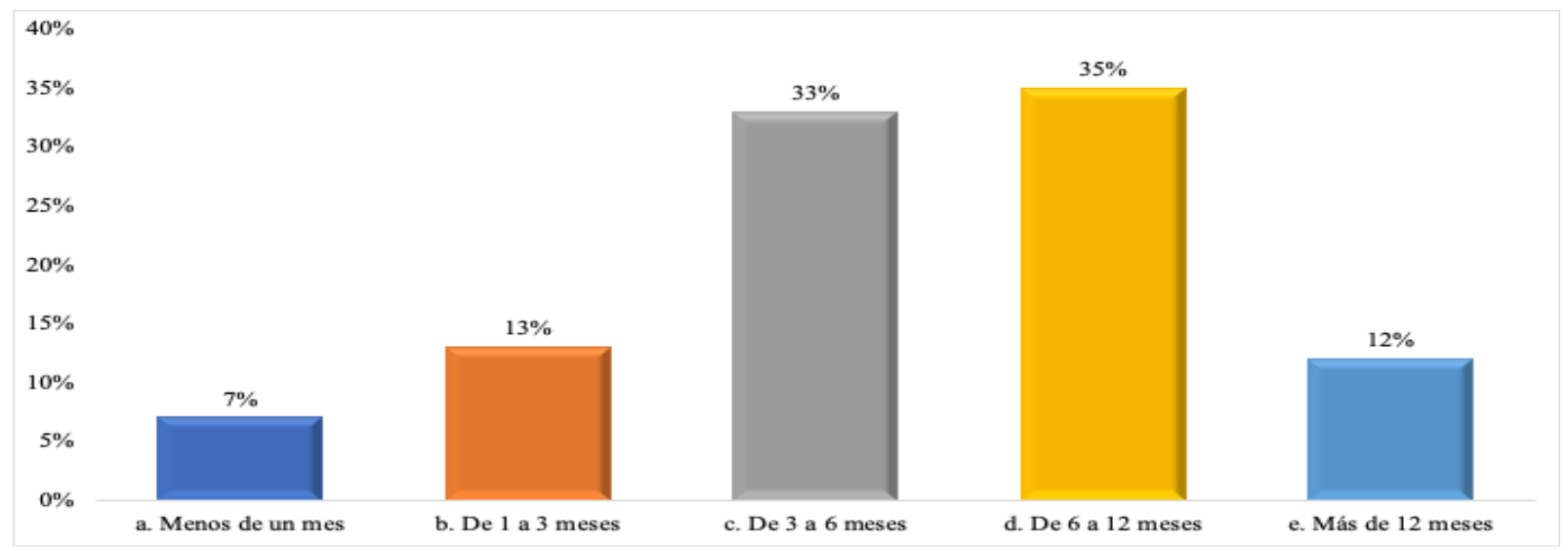

Fuente: Elaboración propia en base a resultados de la investigación.

La imagen 8, muestra que más de una tercera parte de los directores financieros de las Pymes de la muestra analizada estima que le tomará entre 6 a 12 meses retomar sus operaciones normales, a pesar de que el $69 \%$ consideró que a la empresa le tomaría hasta seis meses recuperarse de los efectos de la pandemia. Los resultados obtenidos muestran que el empresario busca rescatar su negocio a un corto plazo, lo anterior hace suponer la importancia que reviste generar estrategias de gestión financiera efectivas para enfrentar la situación de crisis actual.

\section{Discusión de resultados}

Los resultados hacen ver que la situación de las Pymes del sector comercio durante la contingencia generada por el COVID-19 se ha visto afectada por la reducción de la demanda, los incrementos de sus deudas y los cierres de las aperturas de apoyo financiero para poder enfrentarlas. Muchos pequeños empresarios, en su afán de conservar su negocio buscó nuevas y variadas formas de financiamiento para inversión, donde su primera opción fue la propia familia y amigos, lo anterior hace suponer que el empresario fue cauto en medidas de endeudamiento 
porque es consciente de que la rentabilidad de su negocio en momento de crisis no puede sustentar una inversión de momento porque esta esta orientada a aumentar el valor de la empresa y el panorama es aun muy incierto.

\section{Conclusiones}

A pesar de que existen diferentes formas en las que los accionistas pueden financiar el crecimiento de la empresas, la obtención adecuada de recursos económicos es un tema que impacta directamente en su desempeño, por lo que es imprescindible que toda empresa estructure su crecimiento alineando su estrategia financiera con la madurez de su negocio con el objetivo de no incurrir en riesgos financieros innecesarios que puedan comprometer su futuro. Con un panorama incierto sobre la evolución de la pandemia y sus impactos, no sabemos cuántas mipymes sobrevivirán al embate del COVID-19, ni tampoco como se impulsarán las políticas públicas y mecanismos para ayudar a estas empresas a mejorar su situación financiera, mantenerse en el mercado y crecer en el corto y mediano plazo.

La gestión financiera para las Pymes se traduce en la manera en estas empresas emplean sus recursos de manera eficiente para generar valor a través de cómo capitalizar el negocio, dónde adquirir los recursos y cómo lograr que los accionistas obtengan los beneficios deseados. Los resultados de la investigación que aquí se presentan, dan validez a los resultados obtenidos previamente en la etapa exploratoria y, al momento de contrastarlo con la situación actual del impacto de la pandemia provocada por el COVID-19, nos permite probar el objetivo de la investigación y determinar la importancia que tiene la gestión financiera en las Pymes, como una herramienta de control que ayuda a manejar y aprovechar sus recursos económicos para su desarrollo e inversión. Lo anterior pone de manifiesto la viabilidad del presente proyecto de investigación, definiendo con los resultados aquí presentados, la importancia de crear e implementar dentro de la gestión financiera de sus negocios, estrategias que les ayudan lograr el desarrollo e inversión de sus negocios y considerando la situación actual que se vive, es importante centrarse en tres aspectos: el capital de trabajo, el pasivo circulante y la gestión eficiente del efectivo de caja.

A modo de conclusión final y tomando en cuenta los plantemientos anteriores, se propone trabajar como una estratégia de gestión financiera para las Pymes de Hermosillo, Sonora que tome en consideración que para aumentar la liquidez de la empresa es necesario llevar a cabo tres 
Año 13.

Núm. 34 especial. Tema COVID 19.

ISSN: 2007-8870

https://revistainvestigacionacademicasinfrontera.unison.mx/index.php/RDIASF

Recibido el 14 de abril de 2020. Dictaminado mediante arbitraje favorablemente 2 de septiembre de 2020.

acciones fundamentales: reducir el inventario, tratar de cobrar lo antes posible y retrasar los pagos siempre y cuando eso no suponga pagar comisiones o renunciar a descuentos por pronto pago. La realización de este proyecto se considera viable ya que Sonora, cuenta con un sector económico muy dinámico que podría ayudar en la economía del estado, porque la fragilidad financiera de las pymes en escenarios de crisis generalizada requiere el apoyo extraordinario del Estado, para garantizar la continuidad de la actividad productiva como también el mantenimiento de los puestos de trabajo. Este estudio presenta varias limitaciones que sugieren sean trabajos en vías de investigación futuras. La percepción de las estrategias de gestión financieras que utilizan en empresas de mediano y mayor tamaño exitosa, es un resultado que debe analizarse para descartar la relatividad a su tamaño y éxito empresarial. Posteriormente, sería interesante profundizar en los efectos de la correlación entre otras variables que complemente este trabajo y ayude a la mejora del trabajo que realizan las Pymes que le permitan poder enfrentar el mercado actual al que pertenecen.

\section{Referencias}

Banco Interamericano de Desarrollo (2020). Políticas para combatir la pandemia. Informe macroeconómico de América Latina y el Caribe 2020. BID Publicado en Reunión Virtual Washington, D.C., United States of America. Videoconferencia en: https://events.iadb.org/calendar/event/21867?lang=es

Banco Interamericano de Desarrollo (BID, 2019). Brecha financiera de las MYPYMES. Publicado en: https://www.smefinanceforum.org/data-sites/msme-finance-gap

CEPAL. (2020). América Latina y el Caribe ante la pandemia del COVID-19. Efectos económicos y sociales. Naciones Unidas. Informe especial COVID-19, 15. Recuperado de https://repositorio.cepal.org/bitstream/handle/11362/45337/4/S2000264_es.pdf. 
Ferraro, C. y Goldstein, E. ( (2011). Políticas de acceso al financiamiento para las pequeñas y medianas empresas en América Latina. CEPAL, Colección Documentos de Proyectos, Santiago de Clile.

FMI. (2020). Contracción de la Economía Mundial: COVID-19. ProQuest. Recuperdado de EFE News

Service: https://searchproquestcom.ucatolica.basesdedatosezproxy.com/docview/2389172096/B22 1E2F996904BACPQ/ 13? accountid=45660

Gasparini, C., Molinari, A. y Patrucchi, L. (2020). Los organismos internacionales de crédito frente a la crisis del COVID-19 en América Latina: ¿qué? ¿cómo? y ¿cuánto?. Ec-Revista de administración y economía, (4), 11-28.

Goldstein, E. (2011). El crédito a las pymes en la Argentina: Evolución reciente y estudio de un caso innovador. Santiago de Chile: Naciones Unidas.

González-Díaz, R. R., y Perez, L. A. B. (2015). Análisis financiero empresarial del sector comercio como factor de competitividad través de la lógica difusa. Estrategia, 1(1), 110.

González-Díaz, R. R., Lara, R. J. V., López, R. O. y Hernández-Royett, J. (2016). Tax on advertising and commercial advertising: An analysis from Municipal Tax Management. Globalciencia, 2(1), 20-34.

Herrera, D. (2020). Instrumentos de financiamiento para las micro, pequeñas y medianas empresas en América Latina y el Caribe durante el COVID-1. Documento para discusión No. IDB-DP-771 del Banco Interamericano de Desarrollo. Recuperado de https://publications.iadb.org/publications/spanish/document/Instrumentos-definanciamiento-para-las-micro-pequenas-y-medianas-empresas-en-America-Latina-y-elCaribe-durante-el-Covid-19.pdf

INEGI (2018). Encuesta Nacional sobre Productividad y Competitividad de las Micro, Pequeñas y Medianas Empresas (ENAPROCE) 2018. Recuperado de https://www.inegi.org.mx/contenidos/programas/enaproce/2018/doc/ENAPROCE2018Pre s.pdf 
Año 13.

Núm. 34 especial. Tema COVID 19. https://revistainvestigacionacademicasinfrontera.unison.mx/index.php/RDIASF
Revista de Investigación

Académica sin Frontera

ISSN: 2007-8870

Recibido el 14 de abril de 2020. Dictaminado mediante arbitraje favorablemente 2 de septiembre de 2020.

OECD/CAF (2019), América Latina y el Caribe 2019: Políticas para PYMEs competitivas en la Alianza del Pacífico y países participantes de América del Sur, OECD Publishing, Paris, https://doi.org/10.1787/60745031-es.

OECD/CAF (2019). América Latina y el Caribe 2019: Políticas para PYMEs competitivas en la Alianza del Pacífico y países participantes de América del Sur, OECD Publishing, Paris, https://doi.org/10.1787/60745031-es.

Owen, L. (2020). Five ways the coronavirus is affecting women in Asia. BBC News.

Pantaleón, I. (2020). Así es el reto de las empresas durante la pandemia del Covid-19. Forbes. Recuperado de: https://www.forbes.com.mx/negocios-coronavirus-retos-latampandemia/

Rojas, L. (2017). Situación del financiamiento a Pymes y empresas nuevas en américa latina. Banco de Desarrollo de América Latina (CAF). Editorial Corporación de estudios para Latinoamérica (CEPLAN), Chile.

Urquía, E., Pérez, R., y Muñoz, C. (2011). The impact of Accounting Information Systems (AIS) on performance measures: empirical evidence in Spanish SMEs. The International Journal of Digital Accounting Research, 11, 25-43. DOI: 10.4192/1577-8517-v11_2 


\section{Comité editorial}

Dra. Angélica María Rascón Larios

Universidad de Sonora. México

Dra. María del Rosario Molina González

Universidad de Sonora

Dra. Francisca Elena Rochin Wong

Universidad de Sonora. México

Dra. Lidia Amalia Zallas Esquer

Universidad de Sonora. México

Dra. Beatriz Llamas Arechiga

Universidad de Sonora. México

Dr. Rogelio Barba Álvarez

Universidad de Guadalajara. México

Dra. Rosa María Rincón Ornelas

Universidad de Sonora. México

Dr. Juan Flores Preciado

Universidad de Colima. México

Dr. Amado Olivares Leal. Universidad de Sonora

Universidad de Sonora. México

Dr. Guillermo Velázquez Valadez.

Instituto Politécnico Nacional (IPN) México

Dr. Hugo Nefstalí Padilla Torres.

Universidad Estatal de Sonora. México

Dr. Luis Ramón Moreno Moreno.

Universidad Autónoma de Baja California. México

Dr. Miguel Ángel Vázquez Ruiz.

Universidad de Sonora. México

Dra. Lorena Vélez García.

Universidad Autónoma de Baja California. México

Dra. Pabla Peralta Miranda.

Universidad Simón Bolívar, Barranquilla, Colombia

Mtro. Roberto Espíritu Olmos

Universidad de Colima (FCA Tecomán) Colima 
Año 13.

Revista de Investigación

Núm. 34 especial. Tema COVID 19.

Académica sin Frontera

https://revistainvestigacionacademicasinfrontera.unison.mx/index.php/RDIASF

Recibido el 14 de abril de 2020. Dictaminado mediante arbitraje favorablemente 2 de septiembre de 2020.

Dr. Héctor Priego Huertas.

Universidad de Colima (FCA Tecomán) Colima

Mtra. María Guadalupe Alvarado Ibarra.

Universidad de Sonora. México.

MSc. Celso Germán Sánchez Zayas

Universidad de Camagüey, Ignacio Agramonte Loynaz, Cuba

Dra. María Luisa Quintero Soto

Universidad Autónoma del Estado de México

Dr. Eyder Bolivar Mojica

Universidad Católica, Luis Amigó, Medellin, Colombia

Revisores de Textos en Inglés

Mtro. Renato Encinas

Mtra. Cecilia Guadalupe Martínez Solano

\section{Comité científico}

Dr. Rosendo Martínez Jiménez. Universidad Autónoma Benito Juárez de Oaxaca.

Dr. Hugo Neftalí Padilla. Universidad Estatal de Sonora

Dra. María Teresa Gaxiola Sánchez. Universidad de Sonora.

Dr. José Cesar Kaplan. Universidad Estatal de Sonora.

Dr. Alfredo Islas Rodríguez. Universidad de Sonora

Frecuencia de publicación: semestral / 2 números por año. 
Revista de Investigación Académica sin Frontera (RIASF) con (ISSN: 2007-8870) es un interlocutor internacional de acceso abierto revisado diario en línea en el ámbito del de las Ciencias Económicas Administrativas y Sociales. Su objetivo principal es dar a los trabajos de investigación de calidad. Cubre todas las sub-campos de los campos anteriormente mencionados. Proporciona la plataforma a académicos, estudiantes y profesionales. Sólo pública trabajos de investigación y artículos de revisión inicial. Documento presentado debe cumplir con algunos criterios como, debe ser original, inédita y no estén sometidos a ninguna otra revista.

RIASF es una revista arbitrada / Revisión por pares International. Publicamos documentos sobre una variedad de temas, contextos y estrategias de análisis que examinan la relación entre la rápida evolución para la Sociedad y la tecnología del conocimiento.

REVISTA DE INVESTIGACIÓN ACADÉMICA SIN FRONTERA, Año 13, No. 34 especial, tema COVID 19, Julio - diciembre 2020, es una publicación semestral de investigación científica, editada por la Universidad de Sonora, a través de las División de Ciencias Económicas y Sociales, de la Unidad Regional Sur, Blvd. Lázaro Cárdenas No. 100, Col. Francisco Villa, Navojoa, Sonora, Sonora, México, C.P. 85880. Tel. (642) 42599-54.

https://revistainvestigacionacademicasinfrontera.unison.mx/index.php/RDIASF,

revistaacademicasinfrontera@unison.mx.

Editor responsable: Francisco Espinoza Morales. Reserva de Derechos al Uso Exclusivo: 04-2013121811323700-203 e ISSN: 2007-8870, ambos otorgados por el Instituto Nacional de Derecho de Autor. Inscrita en el Directorio de LATINDEX, con Núm. De folio 20014, folio único 14590. Responsable de la última actualización de este Número, Unidad Informática de la Universidad de Sonora, fecha de la última modificación, 30 de diciembre 2020, indexada a Cite Factor Academic Scientific Journal y Journals Master (IIJIF) y Red Latinoamericana de Revistas Académicas en Ciencias Sociales y Humanidades, (Latín Rev). Las opiniones expresadas por los autores no necesariamente reflejan la postura del editor de la publicación. Se autoriza la reproducción total o parcial de los contenidos e imágenes en la presente publicación siempre y cuando se cuente con la autorización del editor y se cite plenamente la fuente. 


\section{Año 13.}

Revista de Investigación

Núm. 34 especial. Tema COVID 19.

Académica sin Frontera

https://revistainvestigacionacademicasinfrontera.unison.mx/index.php/RDIASF

Recibido el 14 de abril de 2020. Dictaminado mediante arbitraje favorablemente 2 de septiembre de 2020.

Nos complace anunciar que su diario, "Academic Research Journal Withoutborders" (ISSN/EISSN 20078870) fue evaluado positivamente en la indexación Citefactor, ahora la página de la revista está disponible en línea, en caso de cualquier problema.

Journals Master | International Innovative Journal Impact Factor (IIJIF)

\section{Red Latinoamericana de revistas Académicas en Ciencias Sociales y Humanidades}

\section{CiteFactor}
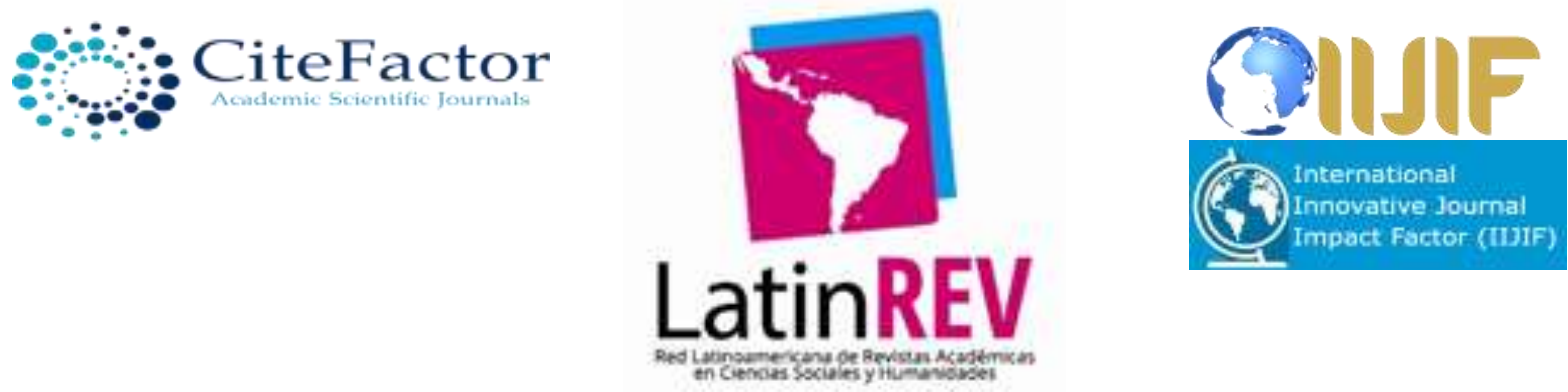

https://www.neliti.com

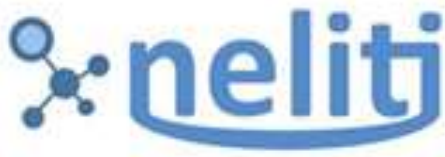

Indonesia's Research Repository
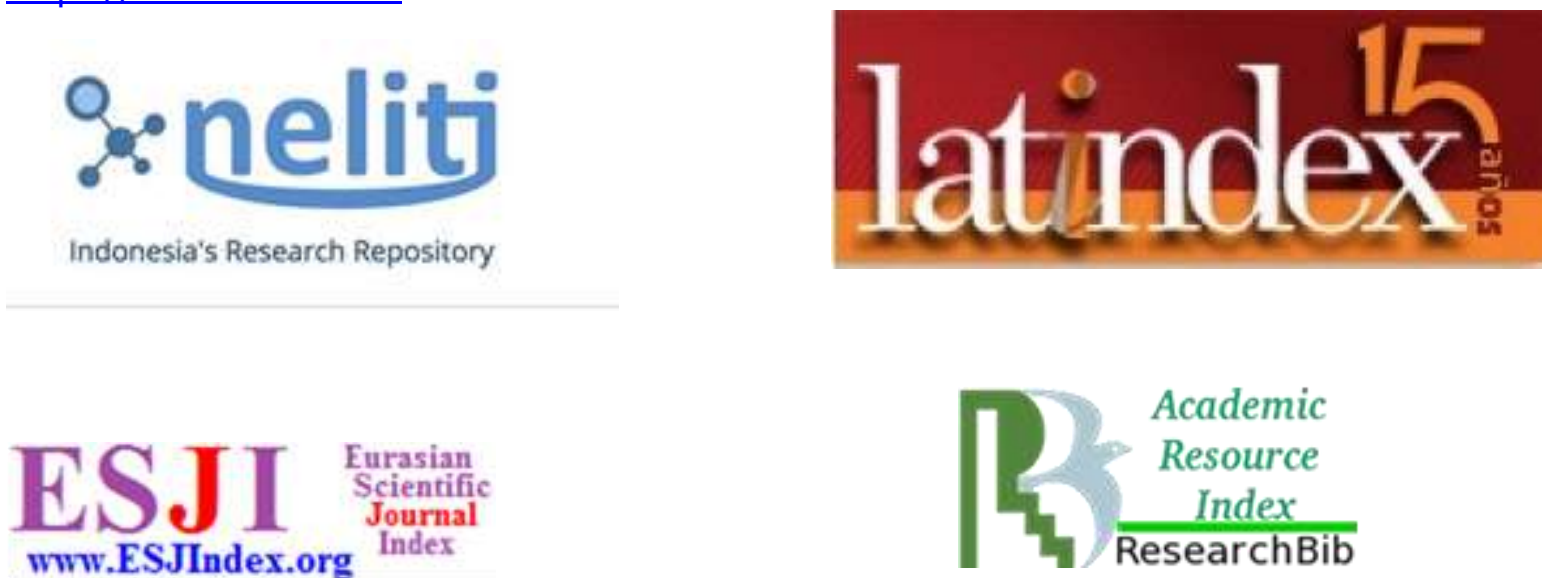